\title{
Stochastic Fluid Model Analysis for Campus Grid Storage Service
}

\author{
Xiaofeng Shi, Huifeng Xue, and Zhiqun Deng \\ College of Automation, Northwestern Polytechnical University, \\ P.O.Box 183\#, Xi' an 710072, China \\ \{Xiaofengshi2002, zhiqundeng\}@tom.com
}

\begin{abstract}
Campus grid storage service is to aggregate the storage resources in the servers of Campus Grid Center and colleges (or institutes, departments), and the storage resources of personal computers in the campus network. It provides storage resources registration, allocation, scheduling, and release services for users by three levels storage architecture. Due to the storage nodes' dynamites, the total storage space that nodes contribute will dynamically change with time. To study the performance of the storage service, the stochastic fluid model is adopted. By this analytical model, we got the mathematical results as follows: the function between the storage allocation probability and the number of nodes is got; if more nodes join the campus grid, the aggregated storage space will be larger, and then the available storage resources will be more; if the storage resources allocation rate is larger than the storage resources release rate, then the available storage resources will decrease.
\end{abstract}

\section{Introduction}

The campus grid is mainly to aggregate all kinds of resources in the campus networks, and provide the services that the campus network currently cannot afford. The Campus grid is not just to provide the High Performance Computing, but also provide other services [1] at a low cost, such as storage service, computing service, and etc.

This paper mainly focuses on the campus grid storage service, which aggregates the storage resources that nodes contribute. Due to nodes' dynamical join and departure, the stochastic fluid model [2,3, and references therein] is adopted to analyze the performance the storage service.

Stochastic fluid model, an important analytic model, has drawn considerable attention in such applications as the performance of P2P cache in [4,5]. In these papers, by this model they got good results from analysis of the cache clusters and P2P cache.

Our work based on [5] is to apply the stochastic fluid model to study the performance of the campus grid storage service. We model the aggregated storage space as a single infinite storage space. Nodes' dynamical join and departure result in the storage space dynamical change, as can be modeled by the stochastic fluid model. Finally, we get the performance of the storage service.

\section{Campus Grid Storage Service Structure}

The campus grid storage service is implemented by three levels architecture (see Fig.1): 


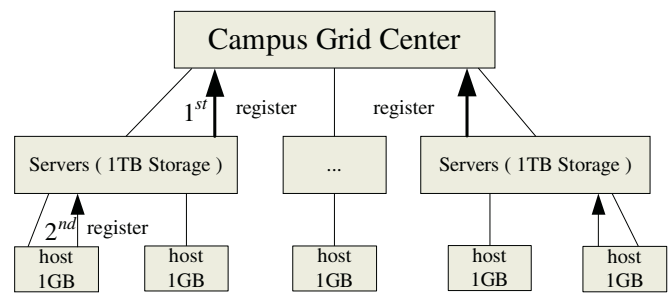

Fig. 1. Nodes' organization architecture of the Campus Grid

1. The first level, Campus Grid Center servers. They manage the registration information of servers in the colleges. The registration information includes the whole storage space, colleges' identifiers, servers' addresses, and etc. Each of these servers contributes at least 1TB storage space.

2. The second level, servers distributed in each college or institute, and department. They manage the registration information of PCs or workstations. Each of these servers contributes at least 1TB storage space.

3. The third level, Hosts (PCs or workstations). They contribute and request the storage resources. They can store data in the campus grid storage space. Each host can contribute $1 \mathrm{~GB}$ or more storage space.

Due to storage nodes dynamical join and departure, Campus Grid Center servers will actively detect the state (work well, temporary stop, stop) of servers in the colleges. Similarly, servers in colleges need to detect the hosts' working state.

\section{General Stochastic Fluid Model for Campus Grid Storage Space}

The whole campus grid system can be viewed as a virtual single storage pool. The total storage space of the universe campus grid system is $c$, which is considered as the total storage space contributed by all the nodes at a given time. $x(t)$ is the total available storage space number (GB). Here, one GB storage space as the minimum unit to contribute and allocate storage resources. If a node joins the system, the storage space number increased is $J(t)$. And if a node leaves the system, the storage space number $(\mathrm{GB})$ reduced is $L(t)$. We define

$\theta=$ Storage resource allocation rate - Storage resource release rate

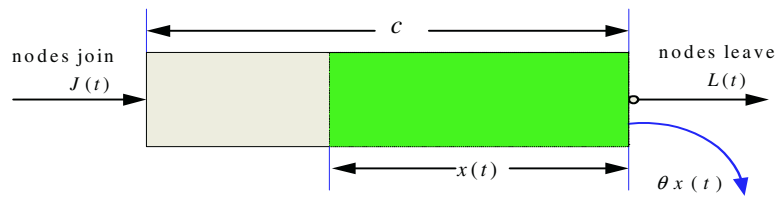

Fig. 2. The general stochastic fluid model for campus grid storage space 
From the above definition, it can be deduced that the storage space number in the system at time $t$ is: $x(t)=J(t)-L(t)-\theta x(t)$. The general stochastic fluid model for campus grid storage space can be seen in Fig.2.

\section{Storage Dynamical Model}

$N$ is the total number of nodes. Nodes go up and down independently of each other, the time until a given up (or down) node goes down (or up) is exponentially distributed. The birth rate (up) of each node is $\lambda$. The death rate (down) is $\mu$. In general, the request of each node randomly arrives based on Poisson processes at a rate $\sigma$.

If there are $i$ nodes that are up, there are $x$ storage spaces that are available. Here $i=0,1, \ldots N$ and $x \in[0, c]$. For each node, the get probability to allocate the storage resources requested is $G$, which can be defined by $G=x / c$.

When a node goes down, the storage space contributed by that node will go down. The remaining number of the storage space in the system is $\Delta_{d}(i) * x$. Here, $\Delta_{d}(i)$ is the down reduction coefficient. Once a node goes up, the storage space will join the system. There is no storage space reduced in the system and the remaining number of the storage space is $\Delta_{u}(i+1) * x$. The up reduction coefficient is $\Delta_{u}(i+1)$. In fact, it can be seen that $0 \leq \Delta_{d}(i) \leq 1$ and $\Delta_{u}()=$.1 .

Duo to the nodes' change, the resulting storage space change is $J(t)-L(t)$, which can be induced by

$$
J(t)-L(t)=i \sigma(1-x / c)
$$

We adopted the simple stochastic fluid model proposed by [5]. Then

$$
G=\frac{E[X]}{c}=\frac{1}{(1+\rho)^{N}} \sum_{i=1}^{N}\left(\begin{array}{c}
N \\
i
\end{array}\right) \rho^{i} v_{i}
$$

Here, the vector $v$ can be seen in [5].

\section{Simulation Analysis}

If we do not consider the effect of storage space allocation and release, then: $\theta=0$. The simulation result can be seen in Fig. 3 as datal curve shows. Else, we consider this, and we set $\theta=0.3$ and $\mu=0.02$. The simulation result can be seen in Fig. 3 as data2 curve shows.

In Fig.3, we can see that the storage allocation probability will be higher if the number of nodes is larger. Then, once users request the storage resources in the campus grid, the probability that the resources are allocated is high. The data 2 curve shows that if the storage resources allocation rate is larger than the storage resources release rate, the probability that the space is allocated will be low. 


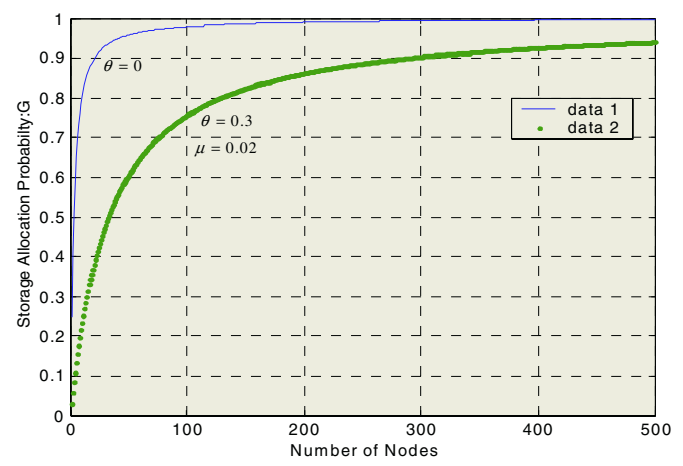

Fig. 3. The relation between number of nodes and the storage resources allocation probability

\section{Conclusions}

In this paper, we have built a general simple virtual storage pool model for the dynamical storage space due to nodes' join and departure. We explore the stochastic fluid analytical models for the purpose of campus grid storage service performance. We have got the results as follows: if more nodes join the campus grid, the aggregated storage space will be larger, and then the available storage resources will be more; if the storage resources allocation rate is larger than the storage resources release rate, then the available storage resources will decrease.

\section{References}

1. Zhiqun Deng, Zhicong Liu, Guanzhong Dai, Xinjia Zhang and Dejun Mu. Nodes' Organization Mechanisms on Campus Grid Services Environment. Lecture Notes in Computer Science, Vol. 3251. Springer-Verlag, Berlin Heidelberg New York (2004) 247-250

2. Nelly Barbot and Bruno Sericola. Distribution of busy period in stochastic fluid models. Communications in Statistics-Stochastic Models, (2001) 17(4)

3. Vidyadhar G. Kulkarni. Fluid Models for Single Buffer Systems, Frontiers in Queuing: Models and Applications in Science and Engineering. Ed. J. H. Dshalalow. CRC Press, (1997) 321-338

4. Florence Clévenot, Philippe Nain. A Simple Fluid Model for the Analysis of the Squirrel P2P Caching System. Proceedings of the IEEE INFOCOM 2004

5. Florence Clévenot, Philippe Nain, Keith W. Ross. Stochastic Fluid Models for Cache Clusters. Performance Evaluation, Vol.59 (1), (2005) 1-18 